Trabajos originales

\title{
Desenlaces del tratamiento con una fórmula extensamente hidrolizada a base de suero en lactantes con alergia a la proteína de leche de vaca
}

\author{
Outcomes of treating infants with cow's milk protein allergies with an \\ extensively hydrolyzed serum-based formula
}

Laura Camila Zuluaga V., ${ }^{1,2}$ Natalia Ramírez R., 1, 2 Lady Katherine Mejía P., 2,3 José Fernando Vera Chamorro. 1, 2,4

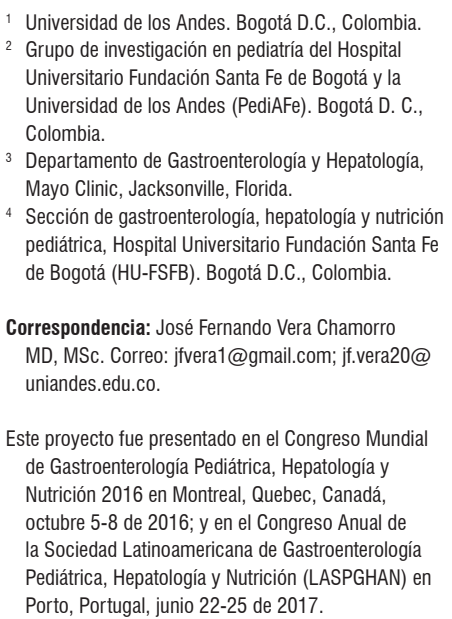

${ }^{3}$ Departamento de Gastroenterología y Hepatología, Mayo Clinic, Jacksonville, Florida.

${ }^{4}$ Sección de gastroenterología, hepatología y nutrición pediátrica, Hospital Universitario Fundación Santa Fe de Bogotá (HU-FSFB). Bogotá D.C., Colombia.

Correspondencia: José Fernando Vera Chamorro MD, MSc. Correo: jfvera1@gmail.com; j.vera20@ uniandes.edu.co.

Este proyecto fue presentado en el Congreso Mundial de Gastroenterología Pediátrica, Hepatología y Nutrición 2016 en Montreal, Quebec, Canadá, octubre 5-8 de 2016; y en el Congreso Anual de la Sociedad Latinoamericana de Gastroenterología Pediátrica, Hepatología y Nutrición (LASPGHAN) en Porto, Portugal, junio 22-25 de 2017.

Fecha recibido: $10-10-17$

Fecha aceptado: 13-04-18

\begin{abstract}
Resumen
Introducción: la alergia a la proteína de leche de vaca (APLV) es la alergia alimentaria más frecuente en el primer año de vida. La evidencia actual acerca de la respuesta a una fórmula extensamente hidrolizada (FEH) a base de suero es insuficiente. Objetivo: determinar los desenlaces clínicos en lactantes con APLV a una FEH a base de suero (Nutrilón Pepti Junior ${ }^{\circledR}$ ). Metodología: estudio retrospectivo, tipo serie de casos. Se incluyeron lactantes ( $\leq 12$ meses) con APLV valorados en la consulta externa entre enero de 2011 y octubre de 2015 en un servicio de gastroenterología pediátrica. La información de datos demográficos, manifestaciones digestivas, tiempo de inicio de síntomas, antropometría y respuesta clínica a la fórmula fue abstraída a partir de la revisión de la historia clínica. Se comparó el estado nutricional de los pacientes en la primera y en la consulta de control un año después utilizando la prueba t de Student. Resultados: se incluyeron 40 lactantes (42,5\% varones) de 3,3 $\pm 2,4$ meses, con síntomas como vómito/regurgitación, cólico/irritabilidad y deposición con sangre. Los antecedentes familiares y perinatales relacionados con el desarrollo de APLV fueron la ingesta de biberón en la primera semana de vida: 27 (67,5\%), madre $\geq 30$ años: 33 (82,5\%), atopia en 2 familiares: $12(30 \%)$ y parto por cesárea: 30 (75\%). $37(92,5 \%)$ pacientes mostraron una respuesta positiva a una $\mathrm{FEH}$ a base de suero, mientras que $3(7,5 \%)$ pacientes no respondieron y requirieron una fórmula a base de aminoácidos (FAA) libres. Los z-score de peso/edad: $-0,69 \pm 1,03$ y peso/talla: $-0,79 \pm 1,00$ de la primera consulta mejoraron en el seguimiento posterior al año $(p<0,05)$ : peso/edad: $-0,27 \pm 0,98$ y peso/talla: $-0,14 \pm$ 0,98 . Conclusión: se encontró recuperación nutricional y tolerancia a la leche de vaca en un alto porcentaje de lactantes con APLV que recibieron una FEH a base de suero.
\end{abstract}

\section{Palabras clave}

Alergia alimentaria, proteína de leche de vaca, lactante, nutrición.

\section{INTRODUCCIÓN}

La alergia a la proteína de leche de vaca (APLV) es la alergia alimentaria más frecuente en el primer año de vida, puesto que la proteína de la leche de vaca (PLV) suele ser la primera proteína a la cual se enfrenta el niño $(1,2)$. Sin un adecuado diagnóstico y tratamiento, el crecimiento y desarrollo psicomotor pueden verse comprometidos, además de predisponer a otras enfermedades atópicas como el asma, dermatitis atópica y rinitis (marcha alérgica); con deterioro de la calidad de vida y aumento en los costos del tratamiento $(1,2)$.

Recientemente, se ha descrito un aumento en la incidencia de las alergias alimentarias, especialmente en países desarrollados $(3,4)$. En el caso de Colombia, múltiples estudios demuestran un posible incremento en la prevalencia de las mismas (5-7). Entre el 5\% y el 15\% de los lactantes muestran síntomas sugestivos de reacciones adversas a las 


\begin{abstract}
Introduction: Cow's milk protein allergies (CMPA) are the most frequent food allergies in the first year of life. Current evidence about responses to an extensively hydrolyzed serum based formula is insufficient. Objective: This study's objective was to determine clinical outcomes in infants with CMPA to an extensively hydrolyzed serum based formula (Nutrilón Pepti Junior $®$ ). Methodology: This is a retrospective case series study of infants 12 months old or younger diagnosed with CMPA in the outpatient clinic of a pediatric gastroenterology service between January 2011 and October 2015. Information including demographic data, digestive manifestations, time of onset of symptoms, anthropometry and clinical responses to formula was abstracted from a review of clinical histories. Patients' nutritional statuses at first and one year follow-up consultations were compared using Student's t-test. Results: Forty infants $(42.5 \%$ males) whose average age was $3.3 \pm 2.4$ months were included. Their symptoms included vomiting/regurgitation, colic/irritability and bloody stools. Family and perinatal history related to the development of CMPA included bottle feeding in the first week of life (27 patients, $67.5 \%)$, mothers who were thirty years of age or older (33 patients, $82.5 \%$ ), atopy in two family members (12 patients, $30 \%$ ) and cesarean deliveries (30 patients, $75 \%$ ). Thirty-seven patients $(92.5 \%)$ responded positively to an extensively hydrolyzed serum based formula while three patients $(7.5 \%)$ did not respond and required a milk-free amino acid based formula. The first consultation z-score of P/E was $-0.69 \pm 1.03$ while the first consultation z-score of $P / T$ was $-0.79 \pm 1.00$. These improved at the one year follow-up to $(p<0.05) P / E:-0.27 \pm 0.98$ and P/T: $-0.14 \pm 0.98$, respectively. Conclusion: Nutritional recovery and tolerance to cow's milk was found in a high percentage of infants with CMPA who received an extensively hydrolyzed serum based formula.
\end{abstract}

\title{
Keywords
}

Food allergy, cow's milk protein, infant, nutrition.

PLV, mientras que las estimaciones de la prevalencia real de la APLV varían del $2 \%$ al $7,5 \%$, cifras que van en aumento en todos los países (8).

La APLV se puede presentar en lactantes alimentados con leche materna o fórmula de leche de vaca y, por lo general, ocurre en las primeras semanas después de la introducción de fórmulas a base de leche de vaca. La APLV se puede encontrar también en lactantes alimentados exclusivamente con leche materna, aunque la incidencia en este grupo es baja $(0,5 \%)$ y la presentación clínica no es tan severa (9). La presentación es variable y ningún síntoma es patognomónico. Las manifestaciones clínicas se producen principalmente en el tracto digestivo (50\%-60\%), la piel (50\%-60\%) y el tracto respiratorio (20\%-30\%); y, según el grado de severidad, se consideran leves, moderadas o graves $(9,10)$.

Se reconoce que algunos antecedentes familiares y perinatales aumentan la predisposición a la alergia, alterando la permeabilidad y la formación de la microbiota intestinal. Dentro de los factores de riesgo descritos se destaca la presencia de atopia familiar, el nacimiento por cesárea, ser madre $>30$ años, ser madre fumadora e inicio de sucedáneo de la leche materna en las primeras semanas de vida (11-21), entre otros.

Una buena historia clínica, incluidos los antecedentes familiares de atopia, la suma de otros factores de riesgo y un examen clínico cuidadoso son la clave en el proceso de diagnóstico de la APLV. Las pruebas cutáneas (leche fresca o extractos de leche entera) de parche y la inmunoglobulina $\mathrm{E}$ (IgE) específicas tienen una sensibilidad del $31,8 \%$ y $90,3 \%$, y especificidad del $20,5 \%$ y $88,9 \%$, respectivamente (22).

Un reto doble ciego controlado con placebo es la regla de oro en el diagnóstico de la APLV; sin embargo, en la prác- tica diaria se realiza un desafío o reto abierto (23). A los lactantes con sospecha de APLV se les retira la PLV durante 2-4 semanas. Aquellos alimentados con fórmula se les suministra una fórmula extensamente hidrolizada (FEH) y se le indica una dieta libre de leche de vaca a la madre lactante, con un suplemento de calcio para evitar complicaciones asociadas. Si existe una APLV, las manifestaciones clínicas desaparecen. A las 4 semanas del retiro se realiza el desafío con la introducción de productos con leche de vaca en la dieta materna en el lactante o fórmulas a base de leche de vaca. La reaparición de los síntomas sugiere, para el presente estudio, el diagnóstico de APLV. Si los pacientes no responden a una $\mathrm{FEH}$, se introducirá una fórmula a base de aminoácidos (FAA).

Un estudio reciente en la población colombiana documentó que durante un período de seguimiento por gastroenterología pediátrica de niños con sospecha de APLV de $14 \pm 1,4$ meses, $47,5 \%$ de los pacientes respondió a una FEH a base de caseína \pm lactancia materna. La mayoría de lactantes sin respuesta presentó una mejoría clínica con una fórmula de aminoácidos \pm lactancia materna y dieta $\sin$ PLV a la madre (21).

No existen estudios en la población colombiana que reporten la respuesta, aceptabilidad y tolerancia a una FEH a base de proteínas de suero de leche de vaca (FEH-S) en pacientes con sospecha de APLV.

\section{OBJETIVO}

Determinar los desenlaces clínicos del tratamiento con una FEH-S (Nutrilón Pepti Junior ${ }^{\circledR}$ ) en lactantes (<12 meses) 
con APLV, atendidos en la consulta externa de la sección de gastroenterología pediátrica de un hospital de referencia.

\section{MATERIALES Y MÉTODOS}

Se realizó un estudio observacional retrospectivo, tipo serie de casos de lactantes ( $\leq 12$ meses) con síntomas sugestivos de APLV que consultaron en la sección de gastroenterología pediátrica del HU-FSFB entre enero de 2011 y octubre de 2015, en quienes se realizó un desafío o reto abierto con una FEH a base de suero (Nutrilón Pepti Junior $\left.{ }^{\circledR}\right) \pm$ leche materna. Para el estudio se tomó como diagnóstico de APLV la reaparición de los síntomas por los cuales consultó el paciente después del desafío con PLV, teniendo en cuenta que no se realizó el reto controlado doble ciego con placebo. Se incluyeron aquellos pacientes con diagnóstico de APLV y que tuvieron seguimiento clínico mínimo de 3 meses después del inicio de la fórmula. Se excluyeron los pacientes con sospecha o diagnóstico principal diferente a APLV (p. ej. síndrome de malabsorción, enfermedades metabólicas, hepáticas, renales, entre otras).

La información de las historias clínicas se recolectó por medio de los sistemas de información HIS-ISIS ${ }^{\circledR}$ del HU-FSFB y MEDSYS V4. $0^{\circledR}$ de la Asociación Médica de los Andes (AMA). La información de: datos demográficos, manifestaciones digestivas, tiempo de inicio de síntomas, antropometría y respuesta clínica a la fórmula se registró mediante un cuestionario consignado en una base de datos en Excel 2010.

El estado nutricional para la primera consulta de gastroenterología pediátrica, segunda consulta y la consulta al año de edad se calcularon de acuerdo con el z-score para peso/edad; talla/edad; peso/talla y perímetro cefálico (PC)/edad con el software OMS Anthro versión 3.2.2. (2011).

Los desenlaces clínicos se definieron de acuerdo con el cambio en la mejoría de los síntomas, la diferencia en el estado nutricional antes y después de la intervención, y la aceptabilidad y tolerancia a la fórmula.

El análisis estadístico se realizó con el programa STATA Special Edition 11.1. Se calcularon medidas de tendencia central para las características demográficas de los pacientes. Se realizaron pruebas t de Student para comparar variables cuantitativas de distribución normal. Se consideró estadísticamente significativo un valor de $p<0,05$. Este estudio fue aprobado por el Comité de Ética Institucional.

\section{RESULTADOS}

Se diagnosticaron 40 lactantes (42,5\% varones) con edad promedio de 3,32 $\pm 2,37$ meses con cuadro clínico de 1,74 $\pm 1,50$ meses de evolución, los cuales se siguieron en la consulta de gastroenterología por 10,65 $\pm 6,76$ meses. Los síntomas que presentaron se resumen en la Tabla 1.
Tabla 1. Cuadro clínico en paciente con APLV $(\mathrm{n}=40)$

\begin{tabular}{lcc}
\hline \multicolumn{1}{c}{ Motivo de consulta } & $\mathbf{n}$ & (\%) \\
\hline Vómito/ERGE & 21 & 52,5 \\
Cólico/irritabilidad & 8 & 20,0 \\
Deposición normal con sangre & 4 & 10,0 \\
Diarrea con sangre & 3 & 7,5 \\
Diarrea sin sangre & 2 & 5,0 \\
Falla del crecimiento & 1 & 2,5 \\
Estreñimiento & 1 & 2,5 \\
\hline
\end{tabular}

ERGE: enfermedad por reflujo gastroesofágico.

El pediatra sospechó APLV solamente en 8 (20\%) pacientes. En los demás pacientes se diagnosticó y trató como ERGE en 21 (52,5\%) pacientes; cólico/irritabilidad en $7(17,5 \%)$, rectorragia en $3(7,5 \%)$ y estreñimiento en 1 (2,5\%) paciente.

Como parte de la valoración y manejo inicial por pediatría, 6 (15\%) pacientes tenían coproscópicos normales, 2 (5\%) hemograma con eosinofilia (>300 eosinófilos totales) y $2(5 \%)$ anemia. En $3(7,5 \%)$ pacientes se descartaron alteraciones estructurales anatómicas mediante una radiografía de vías digestivas altas. 19 (47,5\%) pacientes recibieron terapia con domperidona; $12(30 \%)$ recibieron terapia supresora de ácido con inhibidor de bomba de protones (IBP); 8 (20\%) recibieron bloqueante anti- $\mathrm{H}_{2}$, antiácido y probiótico; 4 (10\%) recibieron antiespasmódico; 3 (7,5\%) recibieron polietilenglicol y simeticona; $y$ un paciente recibió antibiótico y medicación homeopática.

Ante la sospecha diagnóstica, el pediatra indicó una dieta materna libre de lácteos en 13 (32,5\%) pacientes, 11 (27,5\%) recibieron fórmulas parcialmente hidrolizadas $\left(\right.$ Confort $\left.^{\oplus}\right)$, en $6(15 \%)$ el pediatra indicó una FEH a base de suero y en $1(2,5 \%)$ paciente se suspendió la lactancia materna. En el caso de $7(17,5 \%)$ pacientes que recibieron fórmulas parcialmente hidrolizadas (Confort ${ }^{\circledR}$ ) o fórmula de PLV, a sus madres se les ordenó una dieta libre de lácteos.

En la primera valoración por gastroenterología pediátrica, $4(10 \%)$ pacientes cursaban con síndrome broncoobstructivo y en el examen físico se documentaron, 11 $(27,5 \%)$ pacientes con dermatitis atópica y $5(12,5 \%)$ con eritema perianal y costra láctea. La valoración nutricional determinó que $5(12,5 \%)$ pacientes se encontraban con desnutrición aguda moderada y $9(22,5 \%)$ pacientes estaban en riesgo de desnutrición aguda. $1(2,5 \%)$ paciente estaba en riesgo de sobrepeso.

Los factores asociados con el desarrollo de APLV, en congruencia con lo descrito en la literatura, se muestran en la Tabla 2.

El manejo por gastroenterología pediátrica se realizó entre las siguientes aproximaciones: continuar con la lac- 
tancia materna con una dieta materna sin PLV con suplementación de calcio y FEH-S (Nutrilón Pepti Junior"), alimentación exclusiva con una FEH-S o paso a una FAA libres por falta de respuesta (Tabla 3).

Tabla 2. Factores asociados con APLV $(n=40)$

\begin{tabular}{lcc}
\hline \multicolumn{1}{c}{ Factor } & $\mathbf{n}$ & $\%$ \\
\hline Madre $\geq 30$ años & 33 & 82,5 \\
Cesárea & 30 & 75,0 \\
$\quad$ Electiva & 8 & 20,0 \\
Exposición temprana a la leche de vaca & & \\
$\quad$ En la primera semana de vida & 27 & 67,5 \\
$\quad$ En las primeras 24 horas de vida & 20 & 50,0 \\
Atopia en al menos 2 familiares & & \\
$\quad$ De primer grado & 12 & 30,0 \\
$\quad$ De segundo grado & 4 & 10,0 \\
Tabaquismo materno & 2 & 5,0 \\
\hline
\end{tabular}

Tabla 3. Conducta nutricional del gastroenterólogo pediatra $(n=40)$

\begin{tabular}{lcc}
\hline \multicolumn{1}{c}{ Conducta nutricional } & n & $\%$ \\
\hline Lactancia materna + FEH de suero & 25 & 62,5 \\
FEH de suero & 15 & 37,5 \\
Cambio de FEH de suero a FAA & 3 & 7,5 \\
\hline
\end{tabular}

21 pacientes que consultaron por ERGE/vómito requirieron procinéticos y terapia supresora de ácido transitoriamente. Solo a 5 pacientes se les solicitó IgE específica (con resultados negativos), a 5 hemograma (3 presentaron eosinofilia y 2 anemia), a 3 se les descartó anomalías anatómicas mediante una radiografía vías digestivas altas y 1 requirió la realización de un procedimiento endoscópico que demostró proctocolitis eosinofílica. La alimentación complementaria se inició a los 5,22 $\pm 0,54$ meses. $17(67,5 \%)$ pacientes toleraron el huevo y $25(62,5 \%)$ el pescado antes del primer año de vida. Adicionalmente, se determinó que 14 (35\%) pacientes cursaron con dermatitis atópica, $4(10 \%)$ con episodios broncoobstructivos y 3 $(7,5 \%)$ con rinitis alérgica.

Se comparó el estado nutricional de la primera consulta de gastroenterología (3,3 $\pm 2,3$ meses) con el estado nutricional al año de edad de 24 pacientes con la FEH-S y se encontró una mejoría en el seguimiento posterior con el z-score de peso/edad, peso/talla y PC/edad. El cambio del z-score para talla/edad no fue estadísticamente significativo (Tabla 4).

En el seguimiento de 24 pacientes después del año de edad, se encontró que $19(79,2 \%)$ pacientes toleraron la PLV entre los 12 y 17 meses, 4 (16,6\%) entre los 18 y 23 meses, y $1(4,2 \%)$ paciente después de los 2 años.
Tabla 4. Estado nutricional (z-score \pm desviación estándar $[\mathrm{DE}])$ en la primera consulta de gastroenterología pediátrica y en el seguimiento posterior al año de edad

\begin{tabular}{lccc}
\hline z-score & $\begin{array}{c}\text { Primera } \\
\text { consulta de } \\
\text { gastroenterología } \\
\text { pediátrica }(\mathbf{n}=40)\end{array}$ & $\begin{array}{c}\text { Seguimiento de } \\
\text { gastroenterología } \\
\text { pediátrica después del } \\
\text { año de edad }(\mathbf{n}=24)\end{array}$ & $\mathbf{p}^{*}$ \\
\hline Peso/edad & $-0,69 \pm 1,04$ & $-0,27 \pm 0,98$ & 0,01 \\
Talla/edad & $-0,14 \pm 1,27$ & $-0,28 \pm 1,18$ & 0,50 \\
PC/edad & $0,21 \pm 1,01$ & $0,94 \pm 0,80$ & 0,0002 \\
Peso/talla & $-0,79 \pm 1,01$ & $-0,14 \pm 0,98$ & 0,001 \\
\hline
\end{tabular}

${ }^{*} \mathrm{p}<0.05$ (prueba t de Student, muestras pareadas).

\section{DISCUSIÓN}

La evidencia actual acerca de la respuesta a una FEH a base de suero es insuficiente. Los hallazgos de este estudio fortalecen la evidencia disponible acerca de los desenlaces clínicos medidos, tanto en mejoría de síntomas como en el estado nutricional de los pacientes tratados con una FEH a base de suero.

Dentro de los principales factores asociados con el desarrollo de APLV (Tabla 2) se encontraron 2 factores modificables: nacimiento por cesárea y exposición temprana a la PLV. El motivo de consulta principal más frecuentemente encontrado fue vómito y regurgitación, seguido de cólico e irritabilidad. Esta información es acorde con lo reportado en la literatura $(9,10)$. Sin embargo, refleja lo poco específico que puede ser el cuadro clínico, además del corto tiempo de evolución de los síntomas, lo cual puede hacer aún más desafiante la sospecha diagnóstica.

Llama la atención que solo en el $20 \%$ de los casos el pediatra sospechó de APLV, por lo que en la mayoría de los pacientes se tomaron conductas nutricionales no indicadas en el manejo de la APLV como el cambio a otras fórmulas que contienen PLV e incluso interrupción de la lactancia materna en 1 paciente. Como resultado, la conducta nutricional tampoco fue la más adecuada: se encontró una prevalencia del $20 \%$ de terapia con medicamentos antiácidos, que no hacen parte del algoritmo de manejo farmacológico de los síntomas de APLV (23). Lo anterior hace evidente el desconocimiento acerca de este tema desde el ámbito de la pediatría y la atención primaria. Por lo anterior, se hace prioritario el desarrollo de una guía de práctica clínica que estandarice el diagnóstico y manejo de la APLV en nuestro medio desde el primer contacto del paciente con el sistema de salud, con el fin de disminuir la carga de morbilidad asociada con la APLV.

La evaluación clínica detallada en la consulta de gastroenterología pediátrica permitió identificar la concomi- 
tancia de diversas manifestaciones atópicas como la dermatitis atópica, los síndromes broncoobstructivos y la rinitis alérgica hasta en el $25 \%$ de los pacientes. Este hallazgo concuerda con lo descrito en la literatura acerca de la relación de la APLV con la marcha atópica en la infancia (20).

Un gran porcentaje de los pacientes refirió una mejoría de los síntomas en la segunda consulta con gastroenterología pediátrica, la cual sucedía en la mayoría de los casos entre 30 y 90 días después del inicio del tratamiento. Durante el seguimiento, 37 de los 40 pacientes tuvieron una respuesta positiva al tratamiento con la $\mathrm{FEH}$, entendida como la desaparición de los síntomas por los cuales consultaron inicialmente. Se observó una recuperación nutricional estadísticamente significativa en los z-score de peso/edad y peso/talla, lo cual sustenta la pertinencia y utilidad de este tipo de fórmula dentro del tratamiento de los pacientes con APLV. Además, en términos económicos, su costo es mucho menor en comparación con una FAA libres, la cual es el siguiente escalón de tratamiento si se presenta persistencia de los síntomas, tal como sucedió con los 3 casos restantes del total de la muestra.

La tolerancia al pescado y huevo se presentó antes del año de edad en la mayoría de pacientes y a la PLV antes de los 18 meses en más de la mitad de los casos.

A pesar de que este estudio demuestra una adecuada respuesta clínica con una FEH a base de suero en aquellos lactantes con sospecha de APLV, su naturaleza retrospectiva no permite establecer causalidad. Se requieren ensayos clínicos controlados doble ciego que permitan establecer de forma prospectiva la causalidad entre la exposición a una FEH a base de suero y la resolución de los síntomas de APLV.

\section{CONCLUSIONES}

Los principales síntomas de los pacientes incluidos en este estudio y por los cuales consultaron fueron vómito/ ERGE, cólico/irritabilidad y deposiciones sanguinolentas. Los principales factores asociados con el desarrollo de APLV identificados en esta población eran modificables. Se encontró una recuperación nutricional y tolerancia a la leche de vaca en un alto porcentaje de lactantes con APLV que recibieron una FEH a base de suero. Los hallazgos de este estudio son congruentes con otros descritos en la literatura mundial.

\section{Fuente de apoyo financiero}

Danone Nutricia.

\section{REFERENCIAS}

1. Venter C, Arshad SH. Epidemiology of food allergy. Pediatr Clin North Am. 2011;58(2):327-49, ix. doi: 10.1016/j. pcl.2011.02.011.

2. Venter C, Pereira B, Grundy J, et al. Incidence of parentally reported and clinically diagnosed food hypersensitivity in the first year of life. J Allergy Clin Immunol. 2006;117(5):1118-24. doi: 10.1016/j.jaci.2005.12.1352.

3. Branum AM, Lukacs SL. Food allergy among U.S. children: trends in prevalence and hospitalizations. NCHS Data Brief. 2008; (10):1-8.

4. Venter C, Pereira B, Grundy J, et al. Incidence of parentally reported and clinically diagnosed food hypersensitivity in the first year of life. J Allergy Clin Immunol. 2006;117(5):1118-24. doi: 10.1016/j.jaci.2005.12.1352.

5. Marrugo J, Hernandez L, Villalba V. Prevalence of selfreported food allergy in Cartagena (Colombia) population. Allergol Immunopathol (Madr). 2008;36(6):320-4. doi: 10.1016/S0301-0546(08)75863-4.

6. Sole D, Mallol J, Camelo-Nunes IC, et al. Prevalence of rhinitis-related symptoms in Latin American children results of the International Study of Asthma and Allergies in Childhood (ISAAC) phase three. Pediatr Allergy Immunol. 2010;21(1 Pt 2):e127-36. doi: 10.1111/j.13993038.2009.00947.x.

7. Sole D, Mallol J, Wandalsen GF, et al. Prevalence of symptoms of eczema in Latin America: results of the International Study of Asthma and Allergies in Childhood (ISAAC) Phase 3. J Investig Allergol Clin Immunol. 2010;20(4):311-23.

8. Vandenplas Y, Koletzko S, Isolauri E, et al. Guidelines for the diagnosis and management of cow's milk protein allergy in infants. Arch Dis Child. 2007;92(10):902-8. doi: 10.1136/ adc.2006.110999.

9. Marina O, Fernandez A, Follett FR, et al. Cow's milk protein allergy: proposed guidelines for the management of children with cow's milk protein allergy. Arch Argent Pediatr. 2009;107(5):459-67. doi: 10.1590/S032500752009000500016.

10. Correa FF, Vieira MC, Yamamoto DR, et al. Open challenge for the diagnosis of cow's milk protein allergy. J Pediatr (Rio J). 2010;86(2):163-6. doi: 10.2223/JPED.1967.

11. Bjorksten B. Genetic and environmental risk factors for the development of food allergy. Curr Opin Allergy Clin Immunol. 2005;5(3):249-53. doi: 10.1097/01. all.0000168790.82206.17.

12. Burr ML, Merrett TG, Dunstan FD, et al. The development of allergy in high-risk children. Clin Exp Allergy. 1997;27(11):1247-53. doi: 10.1046/j.13652222.1997.970912.x; 10.1111/j.1365-2222.1997.tb01168.x.

13. Eggesbo M, Botten $G$, Stigum $H$, et al. Cesarean delivery and cow milk allergy/intolerance. Allergy. 2005;60(9):1172-3. doi: 10.1111/j.1398-9995.2005.00857.x. 
14. Raimondi F, Indrio F, Crivaro V, et al. Neonatal hyperbilirubinemia increases intestinal protein permeability and the prevalence of cow's milk protein intolerance. Acta Paediatr. 2008;97(6):751-3. doi: 10.1111/j.16512227.2008.00746.x.

15. Sánchez-Valverde F, Gil F, Martinez D, et al. The impact of caesarean delivery and type of feeding on cow's milk allergy in infants and subsequent development of allergic march in childhood. Allergy. 2009;64(6):884-9. doi: 10.1111/j.13989995.2008.01931.x.

16. Acevedo Villafane C, Latorre F, Cifuentes L, et al. Influence of breastfeeding and diet in the development of allergies in children. Aten Primaria. 2009;41(12):675-80. doi: 10.1016/j.aprim.2009.04.005.

17. Dioun AF, Harris SK, Hibberd PL. Is maternal age at delivery related to childhood food allergy? Pediatr Allergy Immunol. 2003;14(4):307-11. doi: 10.1034/j.13993038.2003.00063.x.

18. KramerMS, Kakuma R. Optimal duration of exclusive breastfeeding. Cochrane Database Syst Rev. 2002; (8):CD003517. doi: 10.1002/14651858.CD003517.pub2.
19. Metsala J, Lundqvist A, Kaila M, et al. Maternal and perinatal characteristics and the risk of cow's milk allergy in infants up to 2 years of age: a case-control study nested in the Finnish population. Am J Epidemiol. 2010;171(12):1310-6. doi: 10.1093/aje/kwq074.

20. Tariq SM, Matthews SM, Hakim EA, et al. The prevalence of and risk factors for atopy in early childhood: a whole population birth cohort study. J Allergy Clin Immunol. 1998;101(5):587-93. doi: 10.1016/S00916749(98)70164-2.

21. Vera-Chamorro JF, Ramírez A. Síntomas digestivos y respuesta clínica en lactantes con alergia a la proteína de leche de vaca. Rev Chil Pediatr. 2013;84(6):641-9. doi: 10.4067/ S0370-41062013000600007.

22. BeauchampJN, GabouryI,NiA, etal.Solid-foodintroduction in infants diagnosed as having a cow's-milk protein-induced enterocolitis. J Pediatr Gastroenterol Nutr. 2011;52(5):63943. doi: 10.1097/MPG.0b013e318207ef1a.

23. Vandenplas Y, Koletzko S, Isolauri E, et al. Guidelines for the diagnosis and management of cow's milk protein allergy in infants. Arch Dis Child. 2007;92(10):902-8. doi: 10.1136/ adc.2006.110999. 